\title{
Early Detection of Ovarian Cancer with Conventional and Contrast-Enhanced Transvaginal Sonography: Recent Advances and Potential Improvements
}

\author{
Arthur C. Fleischer, ${ }^{1,2}$ Andrej Lyshchik, ${ }^{1}$ Makiko Hirari, ${ }^{3}$ Ryan D. Moore, ${ }^{1}$ \\ Richard G. Abramson, ${ }^{1}$ and David A. Fishman ${ }^{4}$ \\ ${ }^{1}$ Department of Radiology \& Radiological Sciences, Vanderbilt University Medical Center, 37232 Nashville, TN, USA \\ ${ }^{2}$ Department of Obstetrics and Gynecology, Vanderbilt University Medical Center, 37232 Nashville, TN, USA \\ ${ }^{3}$ Cancer Screening Center and Cancer Institute Hospital, Japanese Foundation for Cancer Research, Tokyo, Japan \\ ${ }^{4}$ Division of Gynecologic Oncology, Mount Sinai Medical Center, 10029 New York, NY, USA
}

Correspondence should be addressed to Arthur C. Fleischer, arthur.fleischer@vanderbilt.edu

Received 18 October 2011; Revised 10 January 2012; Accepted 19 January 2012

Academic Editor: Chih-Ming Ho

Copyright (C) 2012 Arthur C. Fleischer et al. This is an open access article distributed under the Creative Commons Attribution License, which permits unrestricted use, distribution, and reproduction in any medium, provided the original work is properly cited.

Recently, there have been several major technical advances in the sonographic diagnosis of ovarian cancer in its early stages. These include improved assessment of tumor morphology with transvaginal sonography (TVS), and detection and characterization of tumor neovascularity with transvaginal color Doppler sonography (TV-CDS) and contrast-enhanced transvaginal sonography (CE-TVS). This paper will discuss and illustrate these improvements and describe how they enhance detection of early-stage ovarian cancer. Our initial experience with parametric mapping of CE-TVS will also be mentioned.

\section{Introduction}

This year in the United States approximately 24,000 women will be diagnosed with ovarian cancer, and there will be approximately 14,000 associated deaths, predominantly from epithelial ovarian cancer (EOC). Worldwide, it is estimated that 204,449 patients with ovarian cancer will be diagnosed this year with an estimated 124,860 disease-related deaths. The incidence of ovarian cancer has been steadily increasing over the past 10 years, with an overall lifetime risk of $1.8 \%$ [3]. Despite improvements in surgical techniques and new chemotherapeutic regimens, the overall survival for women with stage III/IV EOC has remained relatively unchanged (15\%) over the past 40 years [3]. In contrast, women diagnosed with disease confined to the ovary (stage I) require less morbid surgical intervention, may not require adjuvant chemotherapy, have a significantly improved quality of life, and most importantly have an overall 5-year survival approximating $90 \%$ [3]. Unfortunately, $75 \%$ of women continue to be diagnosed with advanced-stage disease. It is thought that accurate diagnosis of EOC at an earlier stage may decrease overall disease-related mortality.

Evaluation of adnexal masses can be performed with several imaging methods, including TVS, computed tomography (CT), magnetic resonance imaging (MRI), and positron emission tomography (PET). CT can detect large adnexal masses but has lower sensitivity for small adnexal masses, especially in thin patients in whom adnexal lesions can be misinterpreted as other pelvic structures or loops of pelvic small bowel. Furthermore, the ability of CT to characterize adnexal lesions as benign or malignant is limited by low inherent tissue contrast, with the notable exception of ovarian dermoids that can be characterized based on the presence of macroscopic fat and/or calcification. MRI offers higher spatial and contrast resolution than CT and can characterize a wider spectrum of adnexal lesions based on magnetic signal properties or enhancement behavior, but accuracy of MRI may diminish for borderline ovarian tumors and small ovarian masses. Compared to TVS, MRI is costly and has limited availability [4]. PET can identify aggressive 
adnexal lesions on the basis of increased fluorodeoxyglucose (FDG) uptake, but it suffers from low specificity for small lesions, noting that normal premenopausal ovaries will demonstrate increased metabolic activity at mid cycle, and a physiological corpus luteum can therefore mimic an aggressive malignancy. The accuracy of PET is also limited secondary to false negatives in borderline ovarian neoplasms [5].

TVS is widely available and offers high-resolution imaging without the use of ionizing radiation. For these reasons, TVS is the initial diagnostic modality of choice for the evaluation of most patients with a pelvic mass. As previously mentioned, TVS has limited sensitivity and specificity for the definitive diagnosis of ovarian cancer because of overlapping morphologic features seen in benign and malignant lesions. Recently, however, significant technologic advances have yielded vast improvements in the sonographic depiction of early-stage ovarian cancer, and these improvements have translated into improved sonographic discrimination of benign from malignant disease in preliminary studies. Combined evaluation of sonographic morphology and CDS forms a set of basic "simple rules" for sonographic distinction of benign from malignant ovarian masses based on the data derived from a European multicenter study which included 1,223 adnexal tumors (sensitivity 93\%; specificity 90\%) [6] (Table 1).

Three-dimensional transvaginal sonography (3D TVS) has improved the morphologic depiction of ovarian cancers beyond the capabilities of traditional TVS. Improvements in transvaginal color Doppler sonography (TV-CDS) have enhanced sonographic assessment of large tumor vascular networks, and contrast-enhanced transvaginal sonography (CE-TVS) now allows for interrogation of tumor microvascularity [7-9]. This paper discusses these newer techniques, specifically CE-TVS, with emphasis on their advantages and areas for potential improvement.

1.1. $2 D$ and $3 D$ TVS. Conventional sonographic criteria for ovarian cancer diagnosis are based on morphological classification of ovarian masses. Ovarian malignancy is unlikely in simple cysts with smooth walls, but presence of a solid mass or solid projections (papillary excrescences) into the cyst cavity significantly increases the risk of malignancy.

Hirai has described the morphologic features on TVS associated with stage I ovarian cancer in a lay-screening population in Japan [1] (Figures 1, 2, and 3). In general, the stage IA ovarian cancers with normal CA-125 were small and had less solid components than stage IA cancers with elevated CA-125. Papillary excrescences typically occur in areas of epithelial neoplasia and can be seen borderline rather than frankly malignant lesions.

Over the last 10 years, the diagnostic accuracy for conventional 2D TVS has been improving [10]. A 1997 study reported that gray-scale sonography identified malignant tumors with a sensitivity of $91 \%$ and a specificity of $84 \%$ [11], while a 2008 study found a sensitivity of $93 \%$ and specificity of $90 \%$ [12]. As the result of these studies, several morphological scoring systems have been developed
TABLe 1: "Simple rules" for sonographic diagnosis of ovarian cancer*.

\begin{tabular}{ll}
\hline Benign & Malignant \\
\hline (1) Unilocular cyst & (1) Irregular solid tumor \\
(2) Solid components $<7 \mathrm{~mm}$ & (2) Ascites \\
(3) Acoustic shadows & (3) Papillary excrescences \\
(4) Smooth multilocular & (4) Irregular multiloculated/solid \\
$<10 \mathrm{~cm}$ & tumor $>10 \mathrm{~cm}$ \\
(5) No color Doppler flow & (5) Very high color content \\
\hline
\end{tabular}

* Timmerman, D, US O/G $31: 681,2008$.

for sonography, including features such as the presence of papillary projections or irregular and/or thick septae. Results of a meta-analysis provide evidence that sonographic techniques that combine gray-scale morphologic assessment with tumor vascularity mapping are significantly better in ovarian lesion characterization than Doppler arterial resistance measurements, color Doppler flow imaging, or gray-scale morphologic information alone [13].

The recent development of 3D-TVS improves the detection of morphologic abnormalities indicative of neoplastic ovarian masses. In particular, small papillary excrescences or focal wall (mural) irregularities can be detected which are associated with epithelial malignant growth in ovarian masses [8]. The recent advent of matrix array transducers/probes may improve visualization of both internal and external wall (capsular) abnormalities, increase comfort for the patient, and increase reproducibility.

1.2. Transvaginal Color Doppler Sonography (TV-CDS). TVCDS provides depiction of the macrovascularity (over $200 \mu$ ) of tumors but does not delineate microscopic (capillary) tumor neovascularity. The vascular network in tumors can be further interrogated using Doppler techniques to indicate the impedance within vessels $[7,8]$. This in turn roughly reflects pressure gradients.

Combining morphologic assessment with TVS with color Doppler features has allowed accurate assessments of whether a mass is benign or malignant by following "simple rules" [6]. Using color Doppler techniques, the overall vascularity was classified as high, low, or intermediate, rather than determining vascular indices such as resistance or pulsatility. In a European multicentered study, it was shown that this paradigm resulted in 90\% sensitivity and 92\% sensitivity [6].

1.3. Contrast-Enhanced Transvaginal Sonography (CE-TVS). Both micro- and macroscopically, tumor neovascularity is characterized as vessels that demonstrate irregular caliber and branching. TV-CDS can only detect flow in relatively large vessels. Microvascular (i.e., capillary) tumor neovascularity can be depicted using microbubble contrast (Figures 4, 5, and 6). On dynamic CE-TVS malignant tumor neovascularity usually demonstrates a higher peak 

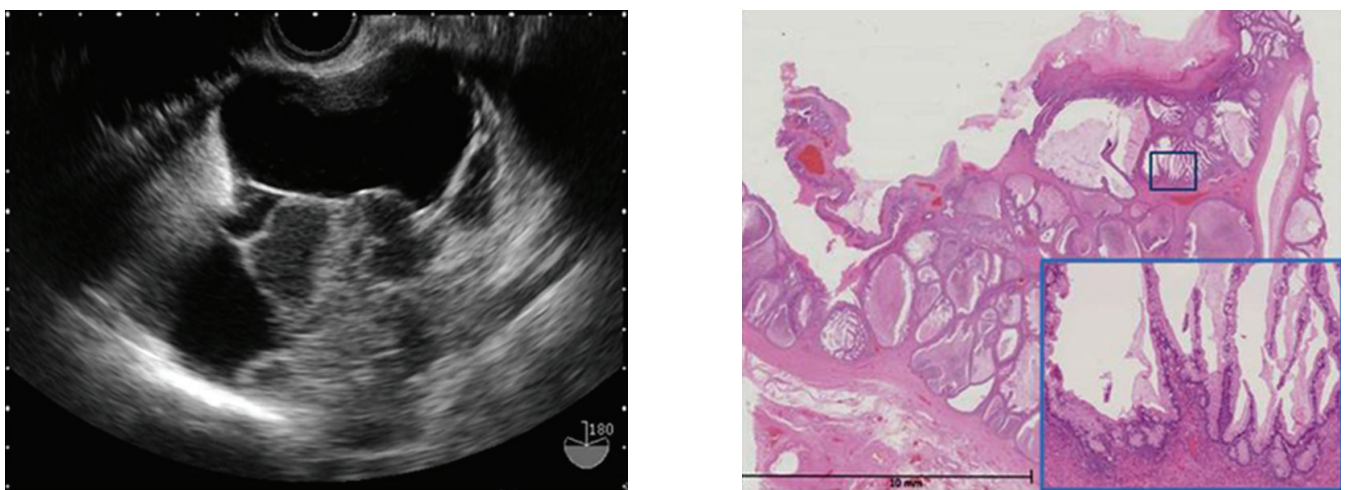

(a) Irregular solid area in a mucinous cystadenocarcinoma
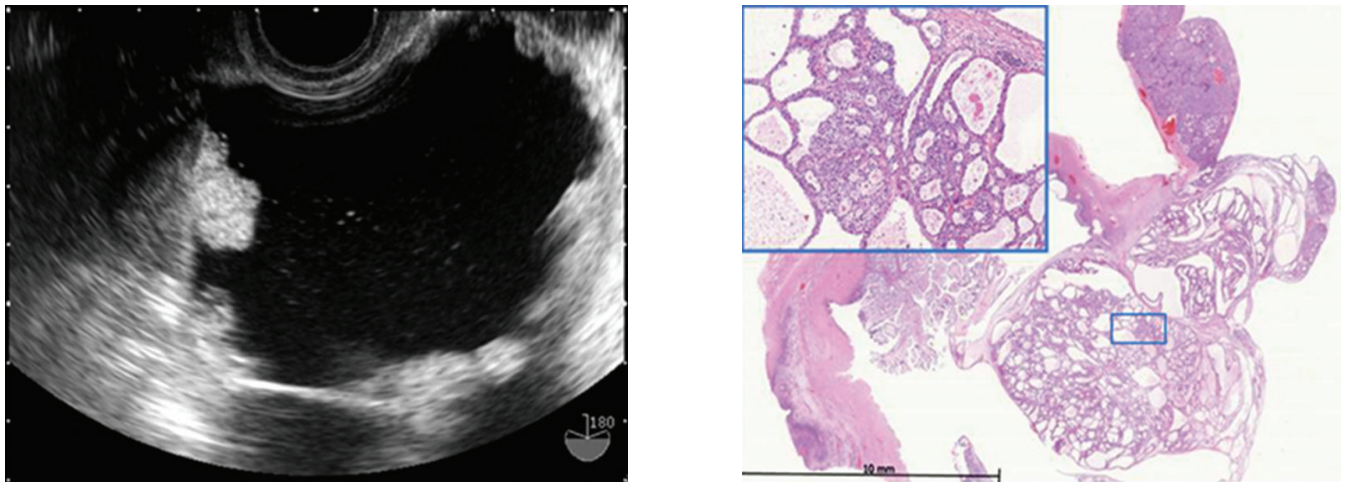

(b) Papillary excrescences in a clear cell carcinoma
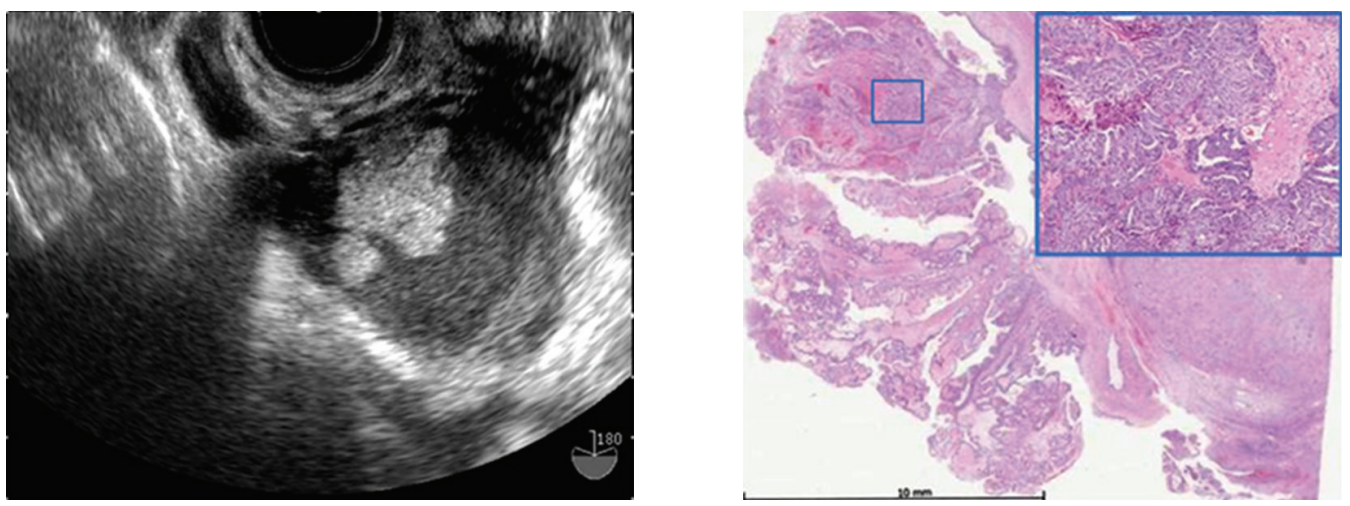

(c) Mural nodules in an endometrioid cancer
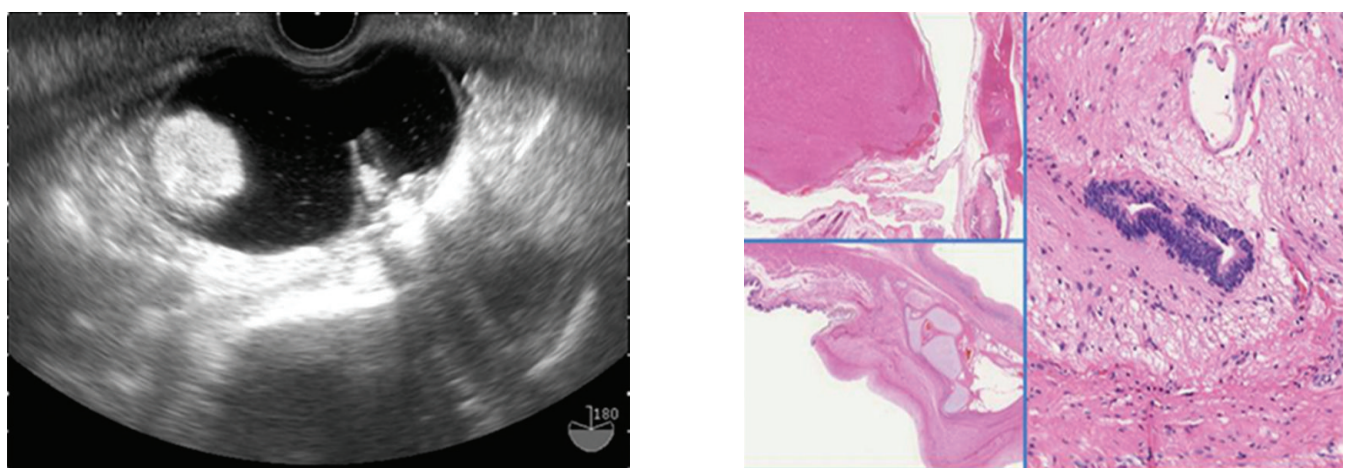

(d) Irregular septa and echogenic solid foci within immature teratoma

FIGURE 1: Morphologic signs of malignancy with histopathologic correlation on TVS in various histologic types of stage 1A ovarian cancer. 

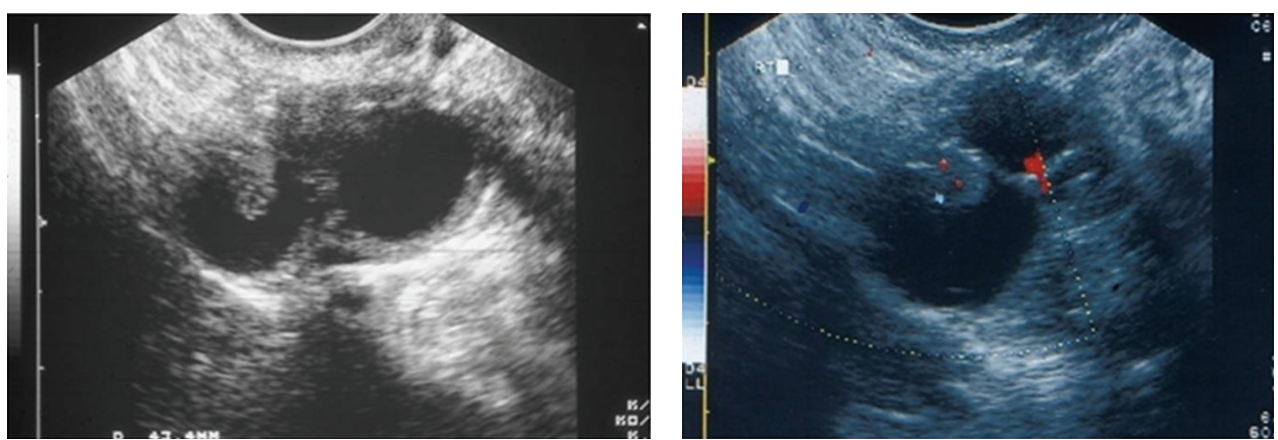

(a) (L) TVS of bilobed cystic ovarian mass containing a papillary excrescence in one locule. (R) CDS showing flow within papillary excrescence
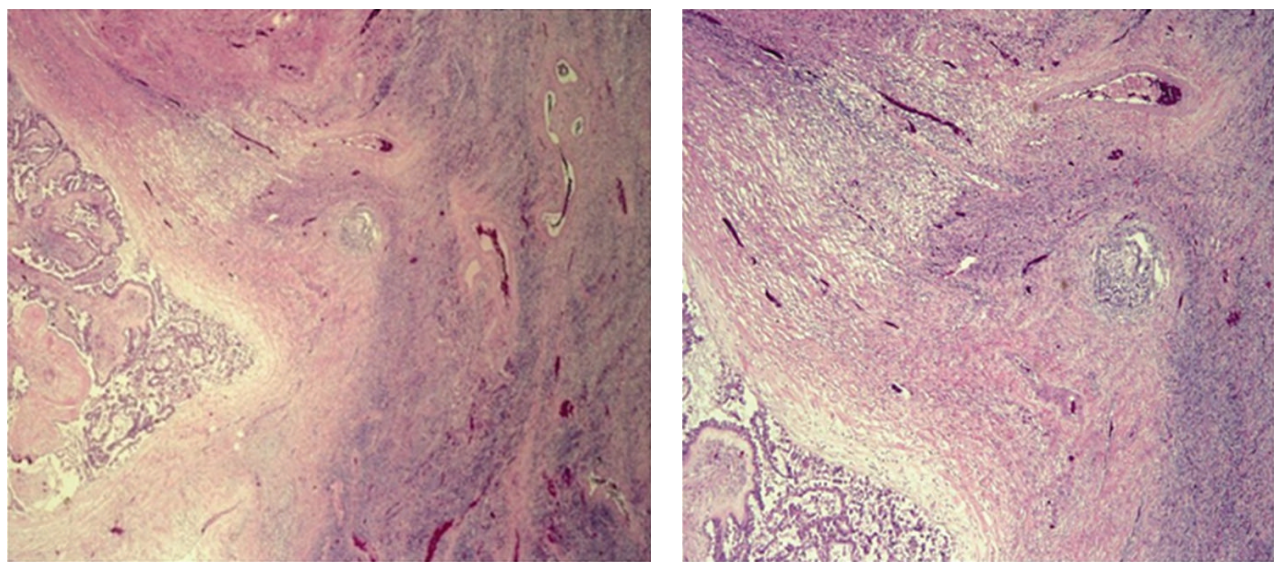

(b) Photomicrographs of histology showing vessels in (L) low power, (R) high power

FIGURE 2: 2D CDS of showing flow within papillary excrescence within a papillary cystadenofibroma.

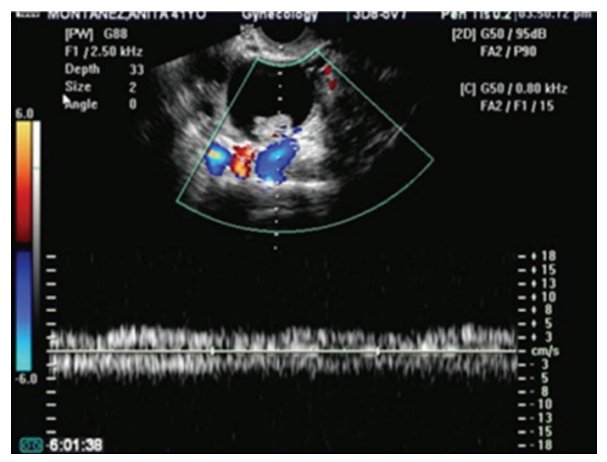

(a) 2D TV-CDS of papillary cystadenoma showing low-impedance flow within a papillary excrescence

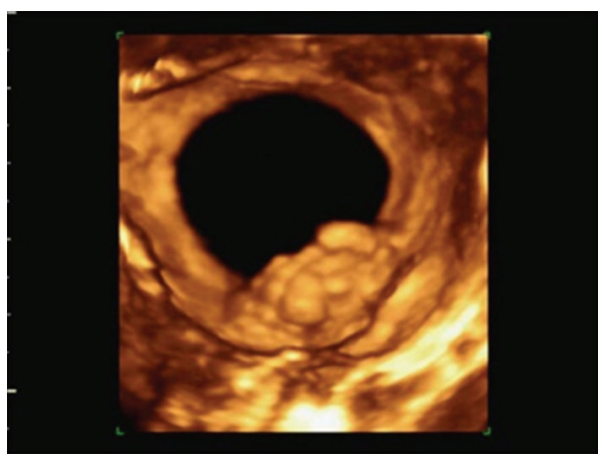

(b) 3D TVS (surface rendition) showing papillary excrescences

FIGURE 3: 3D TV-CDS of papillary excrescences within a papillary serous cystadenoma.

of contrast enhancement and prolonged contrast washout when compared to benign tumors $[1,9,14,15]$ (Figure 9).

In our previous study, all malignant tumors and 50\% of benign tumors showed detectable contrast enhancement (image intensity $>10 \%$ above the baseline) after microbubble injection [9]. When contrast enhancement dynamics were assessed, we found that malignant lesions had a similar time to peak $\left(T_{\mathrm{p}} ; 26.2 \pm 5.9\right.$ versus $29.8 \pm 13.4$ seconds; $P=$ $.4)$, greater peak enhancement $(\mathrm{PE} ; 21.3 \pm 4.7$ versus $8.3 \pm$
$5.7 \mathrm{~dB} ; P<.001)$, a longer half wash-out time $\left((1 / 2) T_{\text {wo }}\right.$; $104.2 \pm 48.1$ versus $32.2 \pm 18.9$ seconds; $P<.001)$, and a greater area under the curve (AUC; $1807.2 \pm 588.3$ versus $413.8 \pm 294.8$ seconds $\left.^{-1} ; P<.001\right)$ when compared with enhancing benign lesions (Figure 9).

AUC greater than 787 seconds $^{-1}$ was the most accurate diagnostic criterion for ovarian cancer, with $100.0 \%$ sensitivity and $96.2 \%$ specificity. Additionally, PE greater than $17.2 \mathrm{~dB}$ (90.0\% sensitivity and $98.3 \%$ specificity) and 

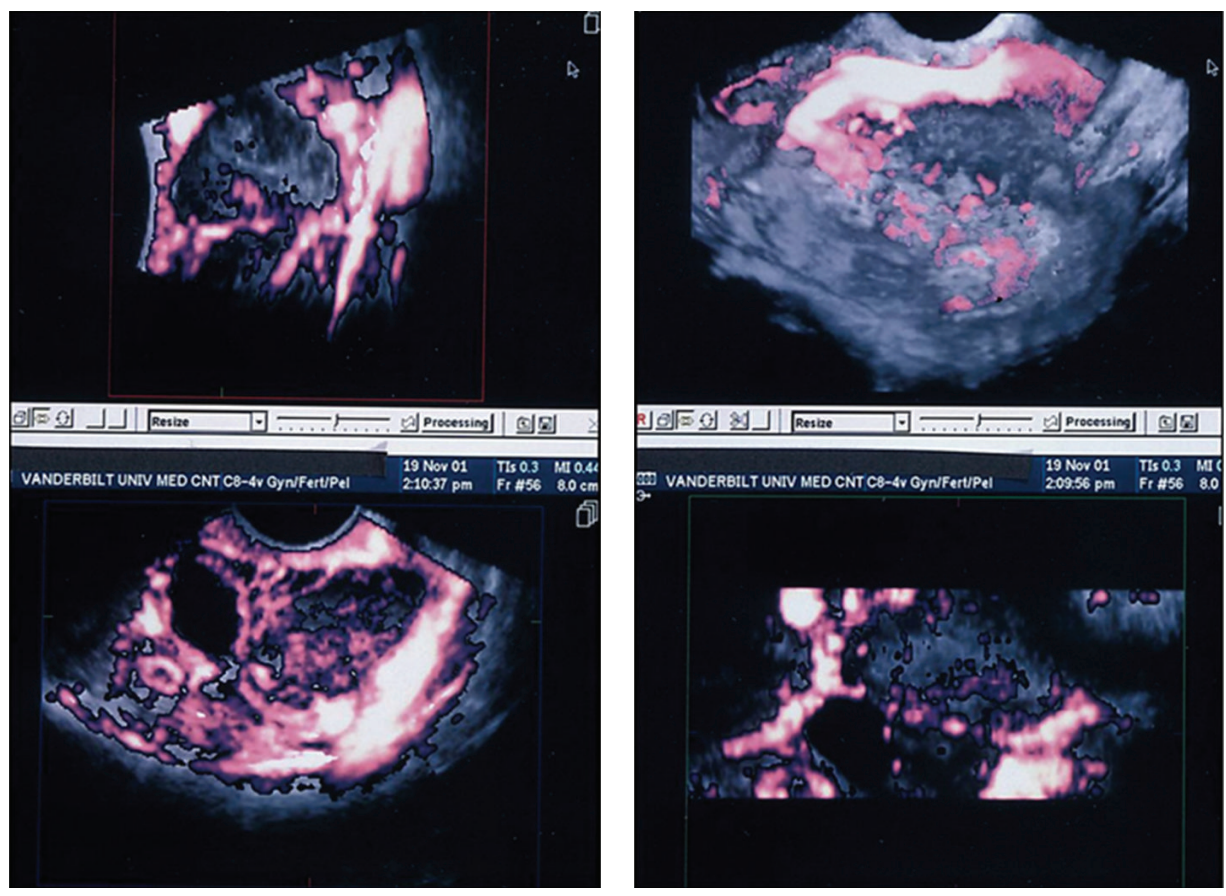

(a) Top: long axis showing central flow with an irregularly shaped solid adnexal mass. Bottom: same as top in short axis

(b) Top: 3D TV-CDS showing (combined volume redition) cluster of vessels within morphologically abnormal area. Bottom: coronal

FIGURE 4: 3D TV-CDS of papillary cystadenocarcinoma showing multiplanar reconstruction (MPR) images.

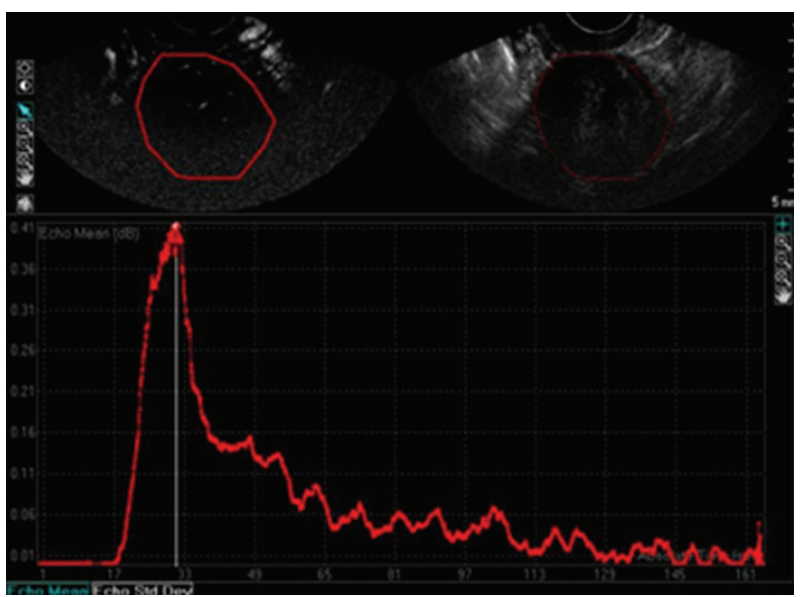

(a) Solid mass with no internal flow with fundamental (top right of image) and harmonic (top left) images. Time intensity curve shows relatively high peak enhancement and short wash-out time

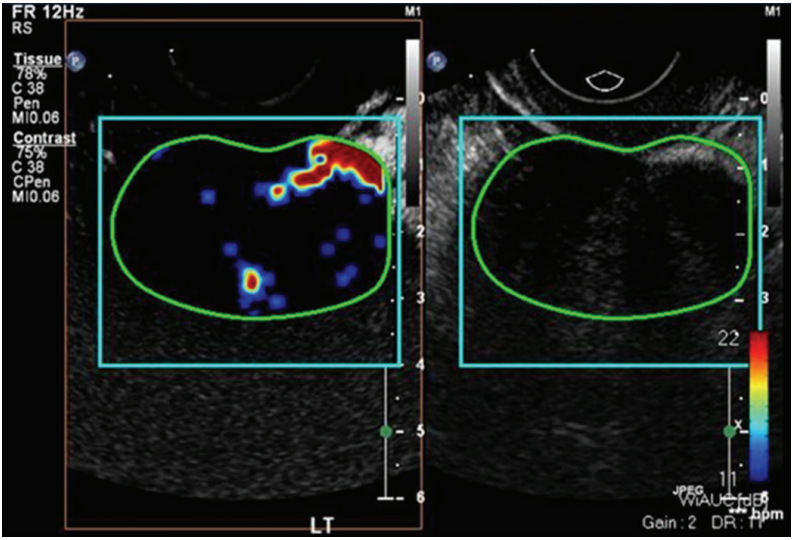

(b) Parametric images showing little internal flow

Figure 5: CE-TVS of a benign fibroma.

a $(1 / 2) T_{\text {wo }}$ of greater than 41.0 seconds (100.0\% sensitivity and $92.3 \%$ specificity) proved to be useful. Initial analysis of contrast-enhanced kinetic was done using time intensity curves for mean, standard deviation, and $P$ value (Figures 9 and 11) and subsequently by receive operator characteristic curves for vascular index (VI); flow index (FI); vascular flow index (VFI) (Figure 12). The receiver operator characteristics of each parameter is shown in Figure 13 with predetermined cutoff values as established with receive operation curves and compared to VI, FI, and VFI values. These results show that contrast-enhanced nonlinear pulse inversion sonography is a more appropriate method for characterizing blood flow dynamics in ovarian tumors than by TV-CDS and can provide an important tool to aid differential diagnoses between benign and malignant ovarian tumors. 


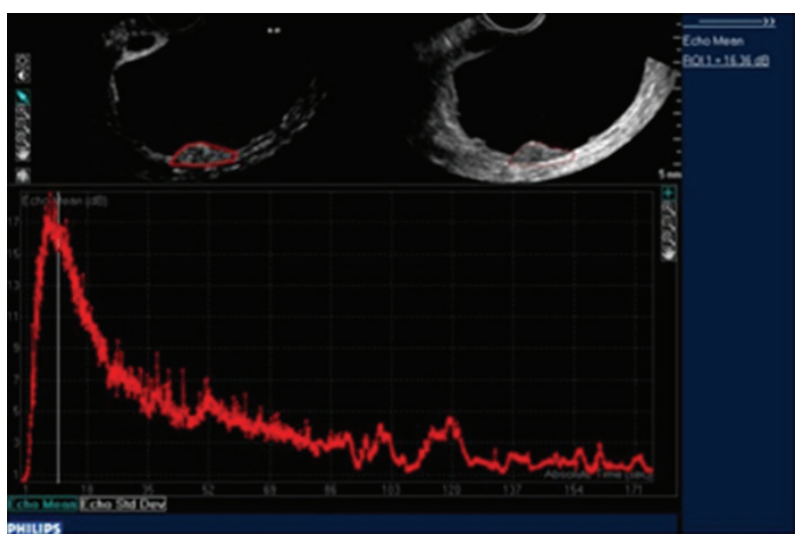

(a) (R) Fundamental and (L) harmonic image showing mural nodule. There is quick wash-in and wash-out within the mural nodule indicating benignancy

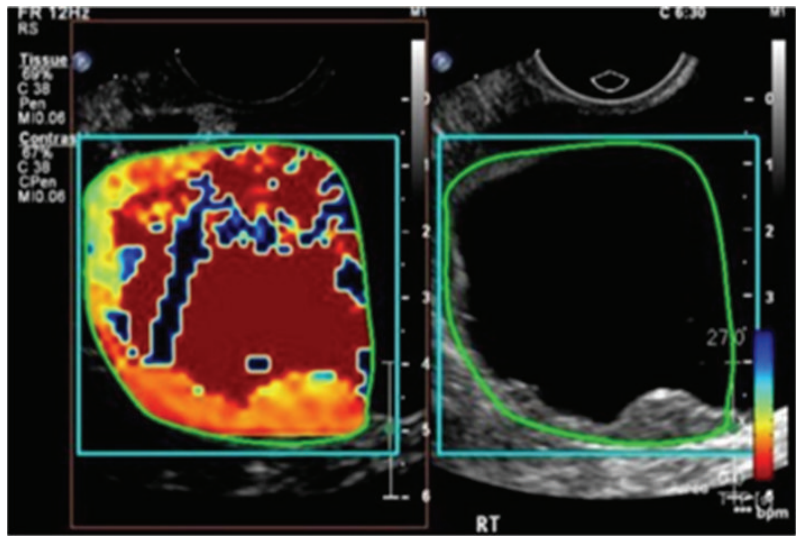

(b) Parametric image showing different time of arrivals within mural nodule, wall, and septum

FIGURE 6: CE-TVS of serous cystadenoma with mural nodules.

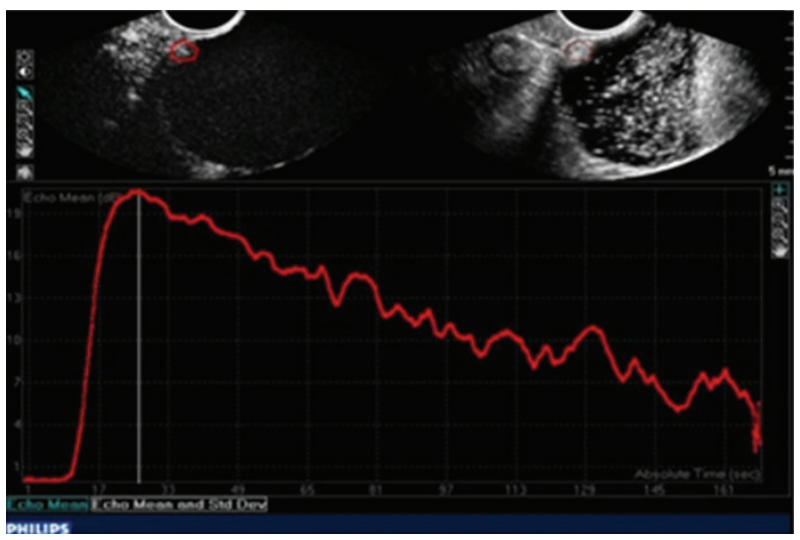

(a) (R) Fundamental image showing mural nodule and mobile echogenic material (L), same using harmonic imaging. There is quick wash-in and long washout within the mural nodule

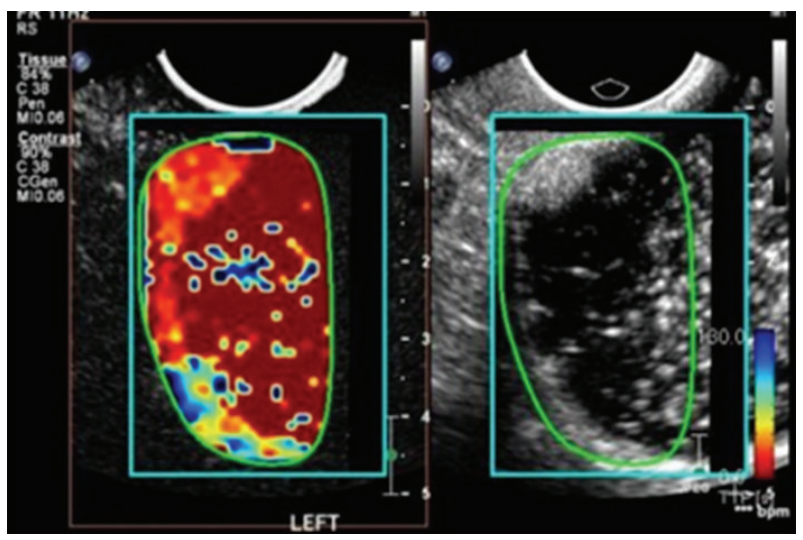

(b) Parametric image different time of arrival for mural module versus wall

FIgURE 7: CE-TVS of borderline mucinous (intestinal) cystadenocarcinoma.

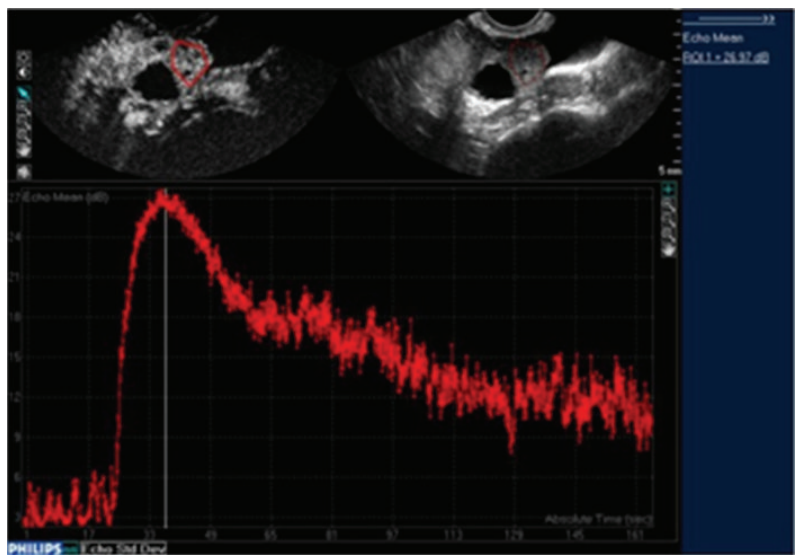

(a) (R) TVS shows normal sized ovary with small cystic area. (L) CDS shows marked vascularity within ovary. Time-intensity curve shows high peak intensity and long washout

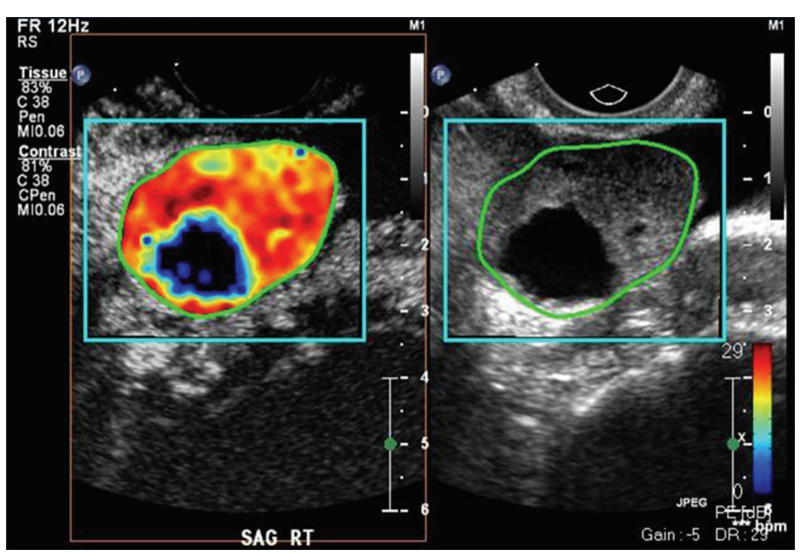

(b) Parametric image showing diffuse vascularity

FIGURE 8: CE-TVS of stage I papillary serous cystadenocarcinoma. 


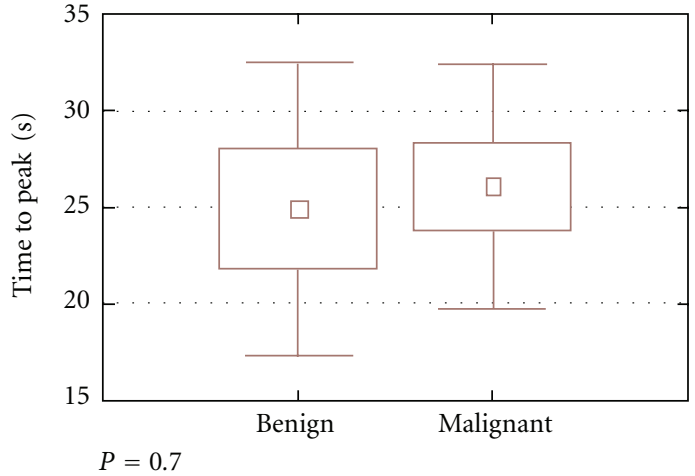

(a)

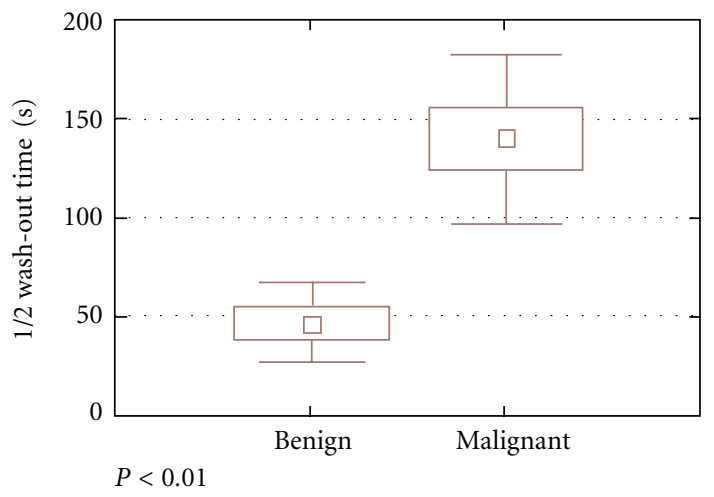

(c)

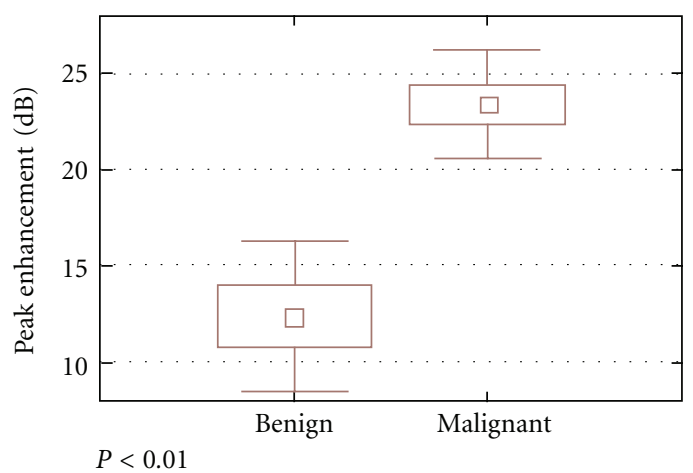

(b)

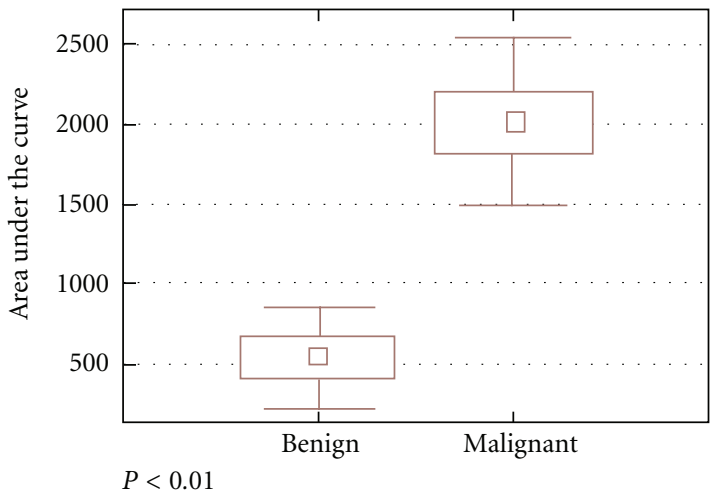

(d)

FIGURE 9: Box graph of contrast-enhanced parameters. While there is no difference in time of peak ( $T$ wash-in), there are significant differences in peak enhancement, wash-out time and vascularity $((\mathrm{b}),(\mathrm{c}) ;(\mathrm{d}))$ from [1].

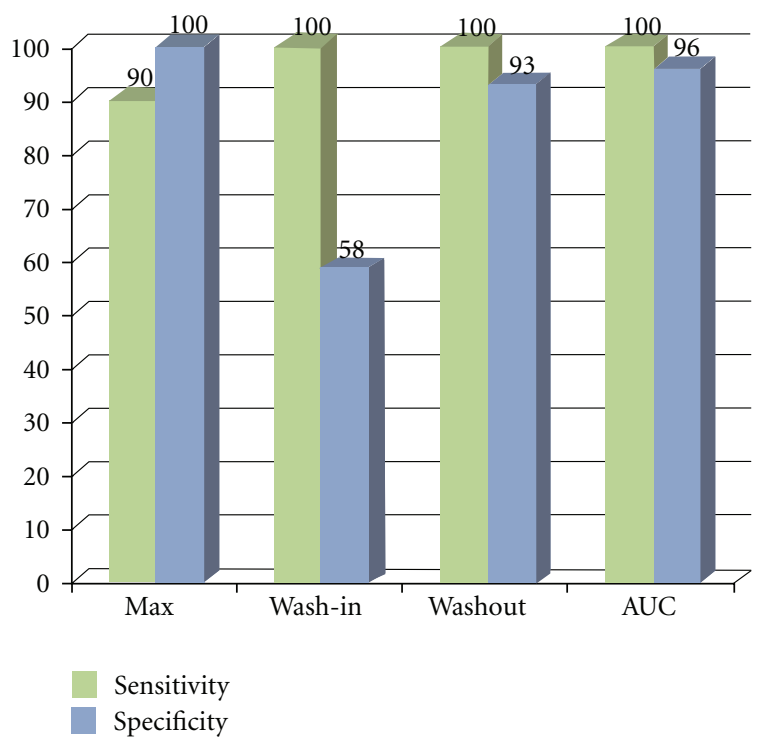

FIGURE 10: Sensitivities and specificities of maximum enhancement, wash-in, wash-out and area under curve (AUC). Maximum enhancement, wash-out and AUC had greatest accuracy.

1.4. Parametric Mapping of CE-TVS. While the timeintensity curve has traditionally been calculated from mean signal intensities over a region of interest, parametric mapping of time-intensity curve variables on a pixel-bypixel basis allows for more global visualization of tumor hemodynamics. The use of this technique in ovarian cancer has been limited to selecting the pixel with greatest peak enhancement (PE) and using that pixel's time-intensity curve for further analysis [15]. However, parametric maps of CE-TVS have recently been used with limited success for differentiation of benign and malignant focal liver lesions [16] and breast lesions $[17,18]$. Preliminary results for using parametric mapping of ovarian tumors seem to indicate significant potential for improving diagnostic accuracy.

Our preliminary results from a subset of 29 out of the 57 subjects analyzed in our previous region of interest (ROI) study show potential for this technique to differentiate benign and malignant ovarian masses [19]. The methods of data acquisition are outlined in the previously described study [9]. Analysis with a quantification software prototype (Bracco Suisse SA, Geneva, Switzerland) utilized parametric maps of $T_{\mathrm{p}}(\mathrm{sec}), \mathrm{PE}(\mathrm{dB})$, and wash-in AUC (wiAUC; arbitrary units, a.u.). The region of interest was kept constant in size between subjects and was corrected for motion. The map color scales were adjusted such that abnormal hemodynamics were represented by red for PE > 24 a.u, $T_{\mathrm{p}}<11 \mathrm{~s}$, and wiAUC $>35$ a.u. (cutoffs chosen at optimum points on receiver operator characteristics curve), and the presence of any red color was used to differentiate benign and malignant tumors. 


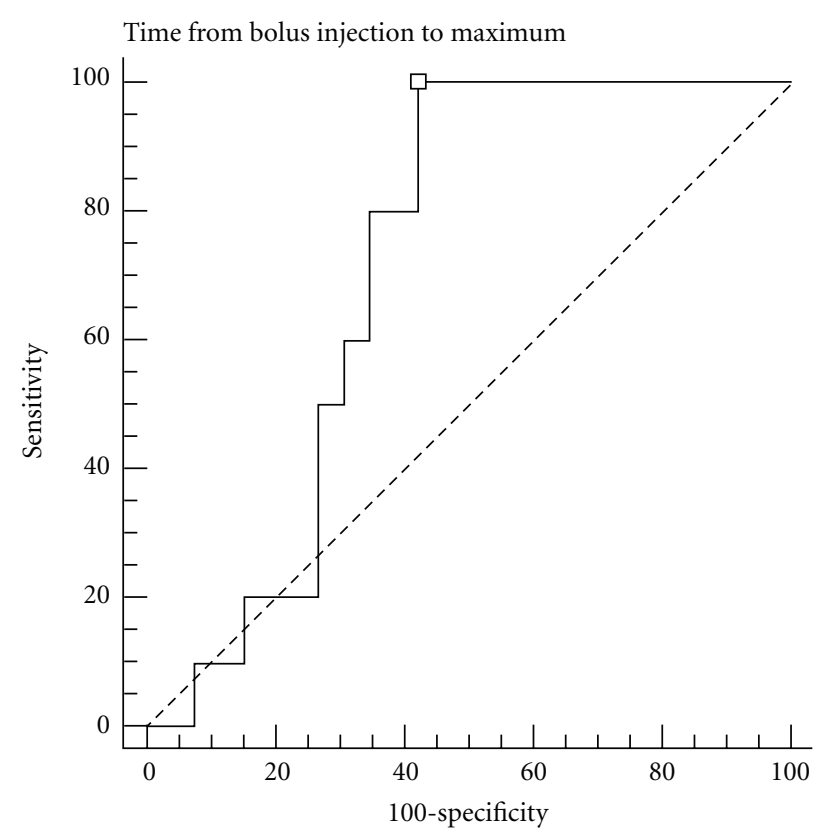

(a)

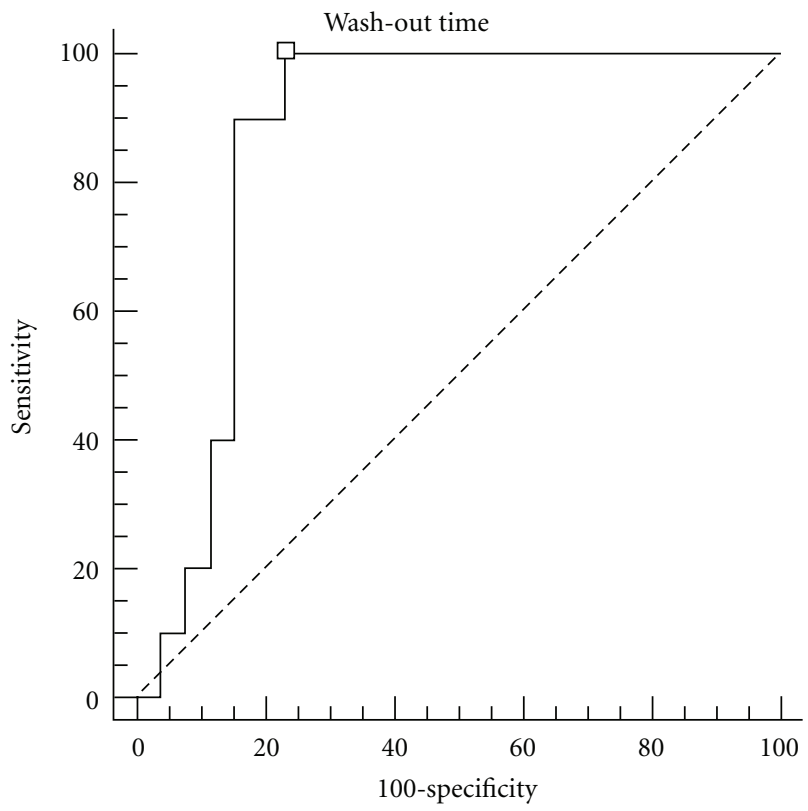

(c)

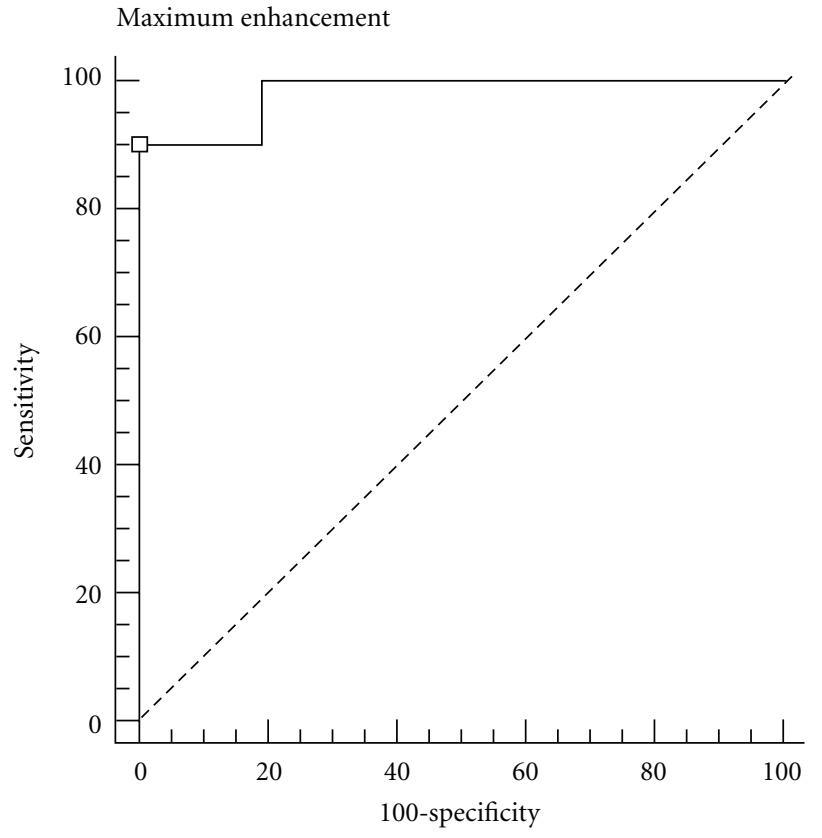

(b)

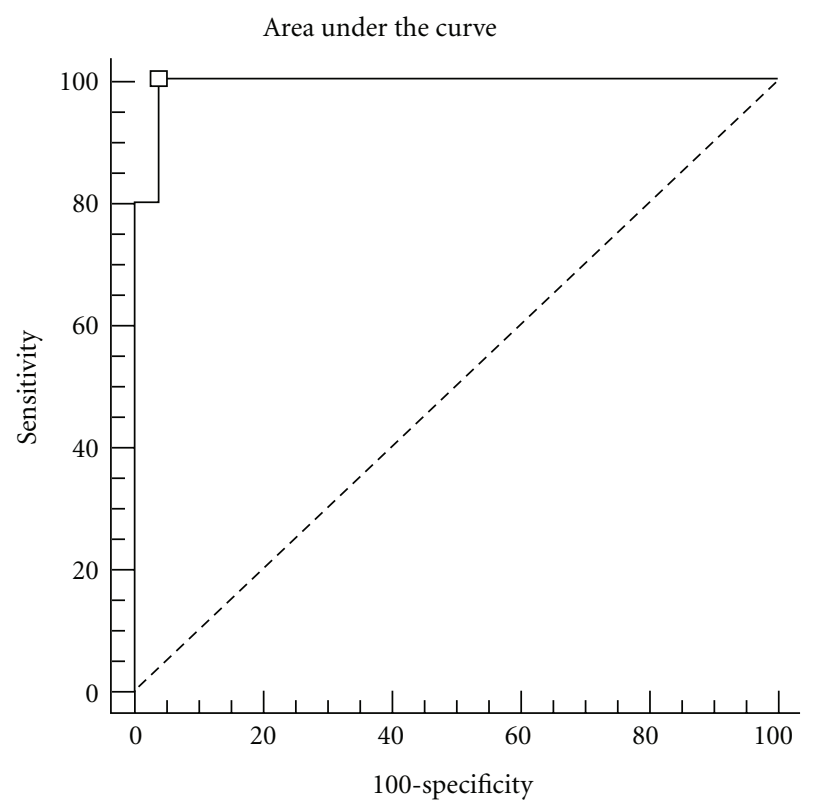

(d)

FIGURE 11: Receiver operator characteristerics for (a) wash-in, (b) maximum enhancement, (c) wash-out, and (d) area under curve.

The preliminary results from the subanalysis of 18 benign and 11 histologically proven malignant ovarian masses showed greatest diagnostic accuracy for maps of PE (sensitivity $100 \%$, specificity $67 \%$ ) and wiAUC (sensitivity $73 \%$, specificity $94 \%$ ), while maps of $T_{\mathrm{p}}$ were least accurate (sensitivity $100 \%$, specificity 17\%). Final analysis of all 57 subjects is needed to determine the ultimate utility of these methods, but preliminary results are promising.

\section{Discussion}

CE-TVS can significantly improve the diagnostic ability of transvaginal sonography alone to identify early microvascular changes that are known to be associated with early-stage ovarian cancer $[1,9,14,15,20]$. Currently, contrast agents play a pivotal role in the imaging modalities of CT and MRI by increasing lesion conspicuity, accentuating morphologic 


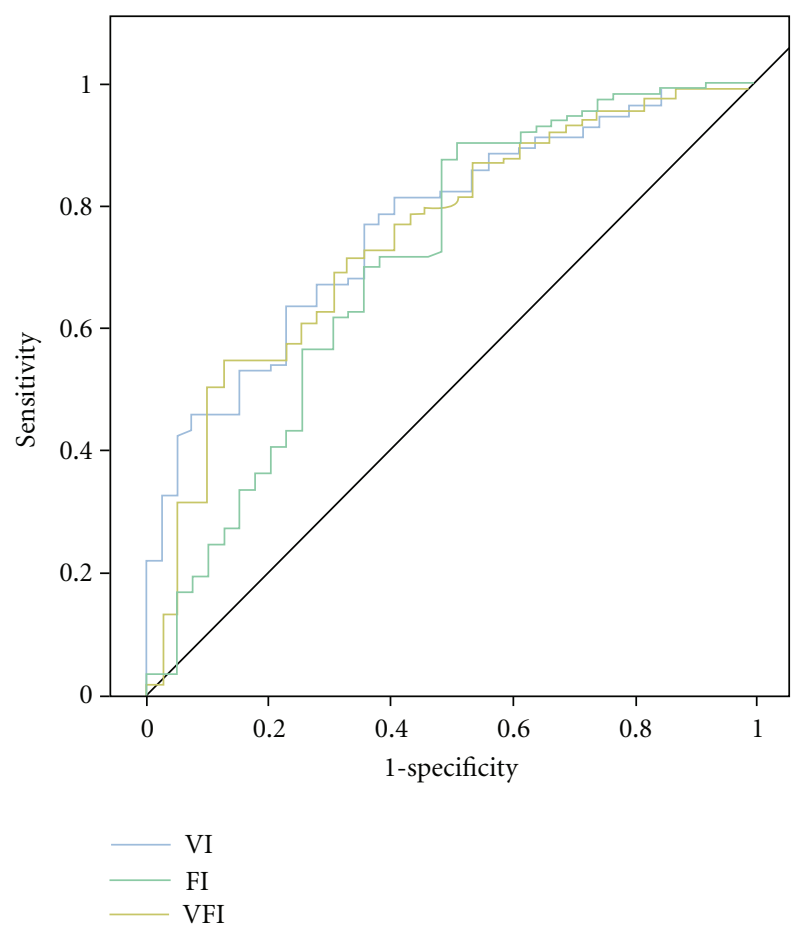

FIGURE 12: Receiver operator characteristic of various parameters showing cutoff points for vascular index (VI), flow index (FI), and vascular flow index (VFI) (from [2]).

features within a lesion, and defining time-resolved lesion enhancement patterns that serve as additional imaging parameters by which a lesion may be characterized. Indeed, contrast agents have received such widespread acceptance that a CT exam performed without intravenous contrast or an MRI without contrast for many indications is now considered limited. Preclinical studies demonstrated that the intravenous contrast agents for sonography hold great promise in a multitude of potential clinical applications, especially in identifying aberrant vascular changes associated with malignancy $[19,21]$.

Previous studies have addressed the use of CE-TVS for benign and malignant tumors by showing greater enhancement of malignant tumors on Doppler imaging. According to the initial work reported by Kupesic and Kurjak, the use of a contrast agent with 3D power Doppler sonography showed very high diagnostic efficiency $(95.6 \%)$ that was superior to that of nonenhanced 3D power Doppler sonography (86.7\%) [22]. However, simple documentation of tumor enhancement may not be sufficient because some benign tumors show detectable contrast enhancement. This limitation can be addressed by assessment of the contrast enhancement kinetics. Only a few studies have been published that used kinetic parameters of the contrast agent to compare benign with malignant tumors in the power Doppler mode. Orden et al. demonstrated that after microbubble contrast agent injection, malignant and benign adnexal lesions behave differently in degree, onset, and duration of Doppler US enhancement.
Comparative techniques

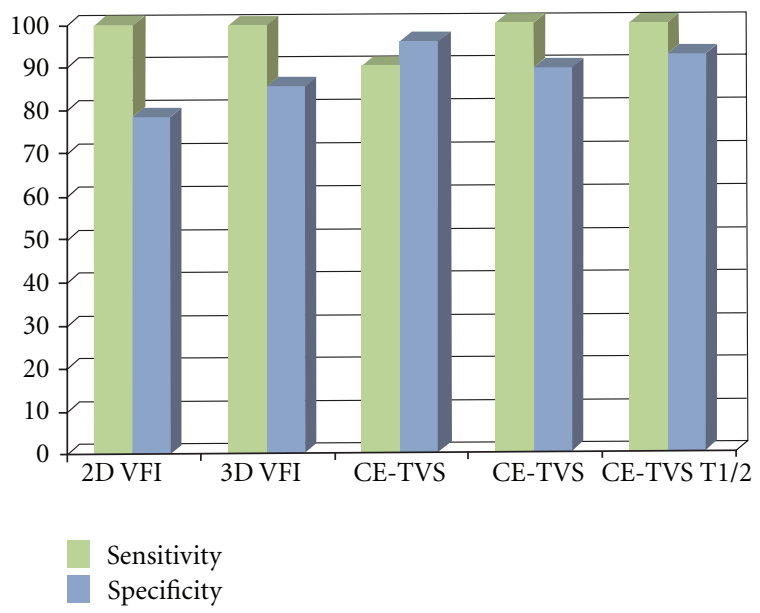

FIGURE 13: Relative accuracy (sensitivity and specificity of enhancement kinetic parameters) of various techniques using predetermined cutoff points of: 2D VFI $(>0.4)$, 3D VFI $(>0.5)$, CE-TVS $(\max >17.2 \mathrm{~dB})$, CE-TVS $\left((1 / 2) T_{\mathrm{wo}}>41 \mathrm{sec}\right)$, CE-TVS (AUC > $\left.787 \mathrm{~s}^{-1}\right)$.

Doppler contrast-enhanced parameters in that study had 79$100 \%$ sensitivity and $77-92 \%$ specificity [23]. Marret et al. reported that wash-out times and AUC were significantly greater in ovarian malignancies than in other benign tumors $(P<.001)$, leading to sensitivity estimates between $96 \%$ and $100 \%$ and specificity estimates between 83 and 98\% [14]. They concluded that Doppler contrast-enhanced parameters had slightly higher sensitivity and slightly lower specificity when compared with transvaginal sonographic variables of the resistive index and serum CA-125 levels [15].

Our preliminary clinical studies explored differences in enhancement parameters in benign versus malignant ovarian masses using a new method of CE-TVS termed pulse inversion nonlinear imaging [9]. This method produces more reliable estimates of tumor microvascular perfusion and provides more consistent results compared to Doppler-based contrast-enhanced ultrasound. Our data suggest that, except for the $T_{\mathrm{p}}$, contrast enhancement parameters are significantly different in benign versus malignant ovarian masses. The $T_{p}$ probably reflects intrinsic circulation depending on cardiac contraction, blood pressure, and overall vascular tone. Once blood circulates through the tumor, however, differences may reflect the unique branching patterns and vessel morphologic characteristics in the microvascularity of the tumors.

As a general statement, contrast enhancement patterns significantly differ between benign and malignant ovarian masses. The addition of a vascular sonographic contrast agent allows a more complete delineation of the vascular anatomy through enhancement of the signal strength from small vessels (capillaries) and provides an entirely new opportunity to time the transit of an injected bolus. CE-TVS has higher sensitivity and specificity to differentiate between benign and malignant lesions than conventional TVS and for detecting occult stage I disease. 
2.1. Future Improvements in CE-TVS. CE-TVS can detect tumor neovascularity (Figures 4, 5, 6, 7, and 8). Tumor neovascularity is characterized by vessels with abnormal endothelial structure that are irregular in caliber and branching patterns. In order to recognize these features, contrast enhancement kinetics show relatively high vascular volume (AUC) and PE. These parameters seem to be best depicted using time-intensity curves. These parameters may also be shown in a parametric map, which allows for stricter cutoff criterion than ROI analysis, as peak values are visualized on a much finer scale than typical ROIs. The traditional approach of calculating the time-intensity curve over ROIs chosen on morphology alone allows small areas of neovascularity to be missed, as they are averaged over larger heterogeneous areas. The primary utility of parametric mapping of ovarian tumors may be guiding selection of ROIs to the area of greatest malignant potential.

With improved imaging technology comes the potential for enhanced therapeutic measures. Specifically, this includes directed therapeutic measures after labeled microbubbles are used [2]. In a murine model this method has been shown to accurately detect sites of active angiogenesis [24]. It is possible that labeled microbubbles could provide directed therapy.

In conclusion, it is hoped that this paper may contribute to the development of new methods for diagnosing, enhancing therapy, and detecting tumor response for this dreaded gynecologic malignancy, possibly with the use of targeted microbubbles [2, 24-28].

\section{Acknowledgment}

Supported in part by National Institute of Health, National Cancer Institute Grant R21 CA 125227-01.

\section{References}

[1] M. Hirai, Y. Hirai, T. Tschida et al., "Comparison of sonographic findings and histopathologic types between patients with normal and elevated serum cancer antigen 125 levels," Journal of Ultrasound in Medicine, vol. 30, no. 7, pp. 943-952, 2011.

[2] L. E. Deelman, A. E. Declèves, J. J. Rychak, and K. Sharma, "Targeted renal therapies through microbubbles and ultrasound," Advanced Drug Delivery Reviews, vol. 62, no. 14, pp. 1369-1377, 2010.

[3] N. Jinawath and I. Shih, "Biology and pathology of ovarian cancer," in Early Diagnosis and Treatment of Cancer. Ovarian Cancer, R. Briston and D. Armstrong, Eds., pp. 17-32, Saunders, Philadelphia, Pa, USA, 2011.

[4] M. Bazot, E. Daraï, J. Nassar-Slaba, C. Lafont, and I. Thomassin-Naggara, "Value of magnetic resonance imaging for the diagnosis of ovarian tumors: a review," Journal of Computer Assisted Tomography, vol. 32, no. 5, pp. 712-723, 2008.

[5] S. Risum, C. Hogdall, A. Loft et al., "The diagnostic value of PET/CT for primary ovarian cancer: a prospective study," Gynecologic Oncology, vol. 105, no. 1, pp. 145-149, 2007.

[6] D. Timmerman, 'Rules' for Sonographic Diagnosis of Ovarian Cancer. US O/G 31:681, 2008.
[7] A. C. Fleischer, J. A. Cullinan, C. V. Peery, and H. W. Jones III, "Early detection of ovarian carcinoma with transvaginal color Doppler ultrasonography," American Journal of Obstetrics and Gynecology, vol. 174, no. 1, pp. 101-106, 1996.

[8] A. C. Fleischer, M. R. Milam, M. A. Crispens, and H. W. Shappell, "Sonographic depiction of intratumoral vascularity with 2- and 3-dimensional color Doppler techniques," Journal of Ultrasound in Medicine, vol. 24, no. 4, pp. 533-537, 2005.

[9] A. C. Fleischer, A. Lyshchik, H. W. Jones et al., "Contrastenhanced transvaginal sonography of benign versus malignant ovarian masses: preliminary findings," Journal of Ultrasound in Medicine, vol. 27, no. 7, pp. 1011-1018, 2008.

[10] L. D. Roman, L. I. Muderspach, S. M. Stein, S. Laifer-Narin, S. Groshen, and C. P. Morrow, "Pelvic examination, tumor marker level, and gray-scale and Doppler sonography in the prediction of pelvic cancer," Obstetrics and Gynecology, vol. 89, no. 4, pp. 493-500, 1997.

[11] A. Reles, U. Wein, and W. Lichtenegger, "Transvaginal color Doppler sonography and conventional sonography in the preoperative assessment of adnexal masses," Journal of Clinical Ultrasound, vol. 25, no. 5, pp. 217-225, 1997.

[12] D. Timmerman, A. C. Testa, T. Bourne et al., "Simple ultrasound-based rules for the diagnosis of ovarian cancer," Ultrasound in Obstetrics and Gynecology, vol. 31, no. 6, pp. 681-690, 2008.

[13] K. Kinkel, H. Hricak, Y. Lu, K. Tsuda, and R. A. Filly, "US characterization of ovarian masses: a meta-analysis," Radiology, vol. 217, no. 3, pp. 803-811, 2000.

[14] H. Marret, S. Sauget, B. Giraudeau et al., "Contrast-enhanced sonography helps in discrimination of benign from malignant adnexal masses," Journal of Ultrasound in Medicine, vol. 23, no. 12, pp. 1629-1639, 2004.

[15] A. C. Testa, D. Timmerman, V. van Belle et al., "Intravenous contrast ultrasound examination using contrast-tuned imaging $\left(\mathrm{CnTI}^{\mathrm{TM}}\right)$ and the contrast medium SonoVue for discrimination between benign and malignant adnexal masses with solid components," Ultrasound in Obstetrics \& Gynecology, vol. 34, no. 6, pp. 699-710, 2009.

[16] A. Anaye, G. Perrenoud, N. Rognin et al., "Differentiation of focal liver lesions: usefulness of parametric imaging with contrast-enhanced US," Radiology, vol. 261, no. 1, pp. 300310, 2011.

[17] J. H. Zhou, W. Zheng, L. H. Cao, and M. Liu, "Contrastenhanced ultrasonic parametric perfusion imaging in the evaluation of antiangiogenic tumor treatment," European Journal of Radiology. In press.

[18] J. R. Eisenbrey, J. K. Dave, D. A. Merton, J. P. Palazzo, A. L. Hall, and F. Forsberg, "Parametric imaging using subharmonic signals from ultrasound contrast agents in patients with breast lesions," Journal of Ultrasound in Medicine, vol. 30, no. 1, pp. 85-92, 2011.

[19] R. D. Moore, A. Lyshchik, and A. C. Fleischer, "Parametric depiction of contrasted sonography of ovarian masses," American Institute of Ultrasound in Medicine. In press.

[20] A. C. Fleischer, A. Lyshchik, R. F. Andreotti, M. Hwang, H. W. Jones III, and D. A. Fishman, "Advances in sonographic detection of ovarian cancer: depiction of tumor neovascularity with microbubbles," American Journal of Roentgenology, vol. 194, no. 2, pp. 343-348, 2010.

[21] K. J. Niermann, A. C. Fleischer, J. Huamani et al., "Measuring tumor perfusion in control and treated murine tumors: correlation of microbubble contrast-enhanced sonography to dynamic contrast-enhanced magnetic resonance imaging and fluorodeoxyglucose positron emission tomography," Journal of Ultrasound in Medicine, vol. 26, no. 6, pp. 749-756, 2007. 
[22] S. Kupesic and A. Kurjak, "Contrast-enhanced, three-dimensional power Doppler sonography for differentiation of adnexal masses," Obstetrics and Gynecology, vol. 96, no. 3, pp. 452$458,2000$.

[23] M. R. Orden, J. S. Juvenlin, and P. P. Kirkinien, "Kinetics of a US contrast agent in benign and malignant adnexal tumors," Radiology, vol. 226, pp. 405-410, 2003.

[24] C. R. Anderson, J. J. Rychak, M. Backer, J. Backer, K. Ley, and A. L. Klibanov, "scVEGF Microbubble ultrasound contrast agents: a novel probe for ultrasound molecular imaging of tumor angiogenesis," Investigative Radiology, vol. 45, no. 10, pp. 579-585, 2010.

[25] M. Hwang, G. Hariri, A. Lyshchik, D. E. Hallahan, and A. C. Fleischer, "Correlation of quantified contrast-enhanced sonography with in vivo tumor response," Journal of Ultrasound in Medicine, vol. 29, no. 4, pp. 597-607, 2010.

[26] J. M. Warram, A. G. Sorace, R. Saini et al., "A triple-targeted ultrasound contrast agent provides improved localization to tumor vasculature," Journal of Ultrasound in Medicine, vol. 30, no. 7, pp. 921-931, 2011.

[27] C. A. Johnson, R. J. Miller, and W. J. O’Brien Jr., "Ultrasound contrast agents affect the angiogenic response," Journal of Ultrasound in Medicine, vol. 30, no. 7, pp. 933-941, 2011.

[28] I. Tardy, S. Pochon, M. Theraulaz et al., "Ultrasound molecular imaging of VEGFR2 in a rat prostate tumor model using BR55," Investigative Radiology, vol. 45, no. 10, pp. 573-578, 2010. 


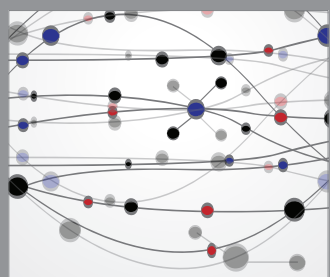

The Scientific World Journal
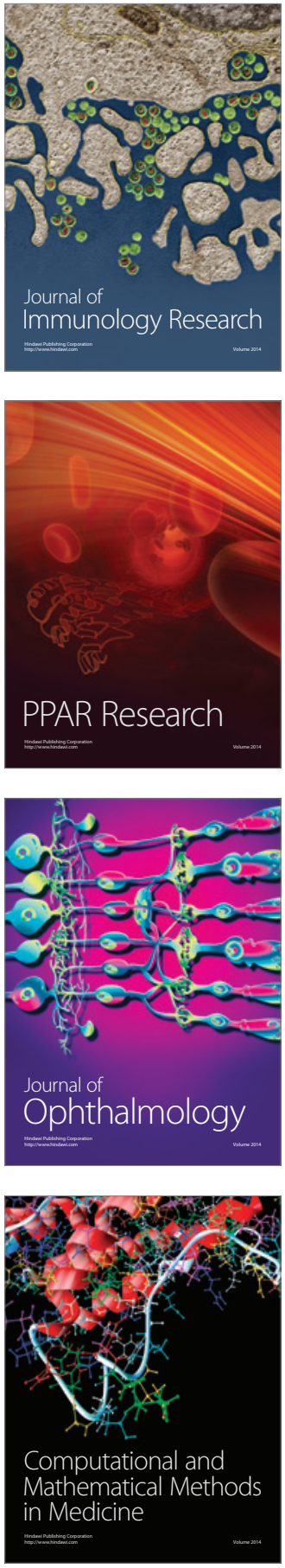

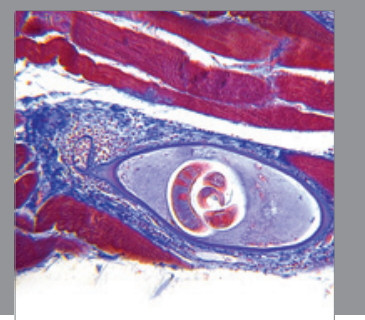

Gastroenterology

Research and Practice
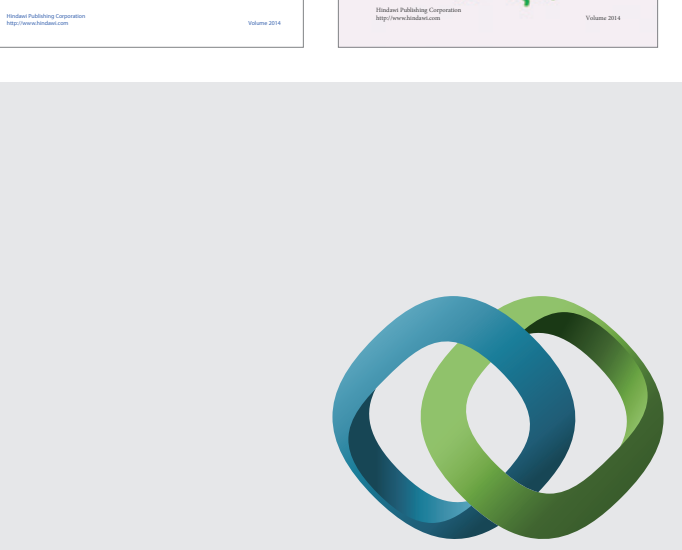

\section{Hindawi}

Submit your manuscripts at

http://www.hindawi.com
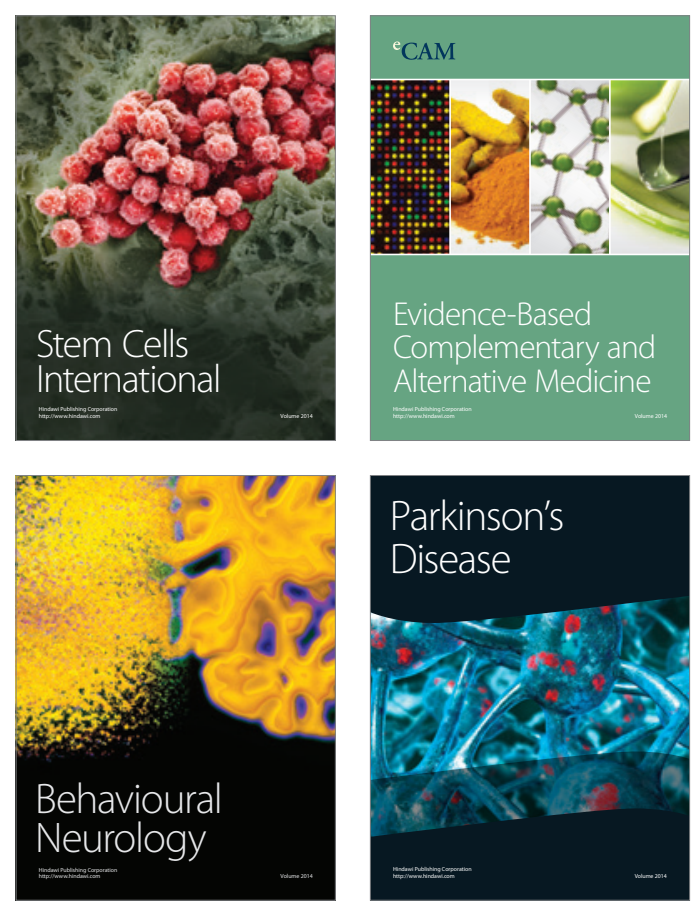

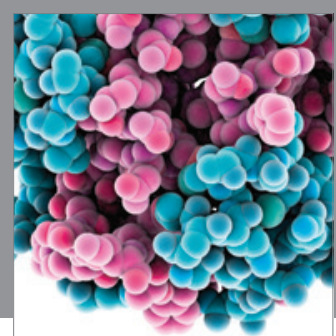

Journal of
Diabetes Research

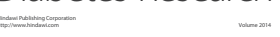

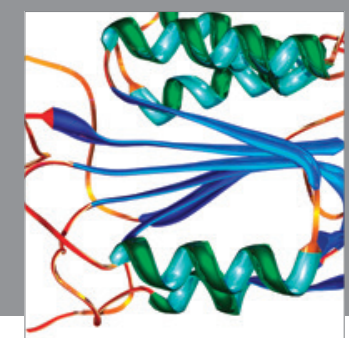

Disease Markers
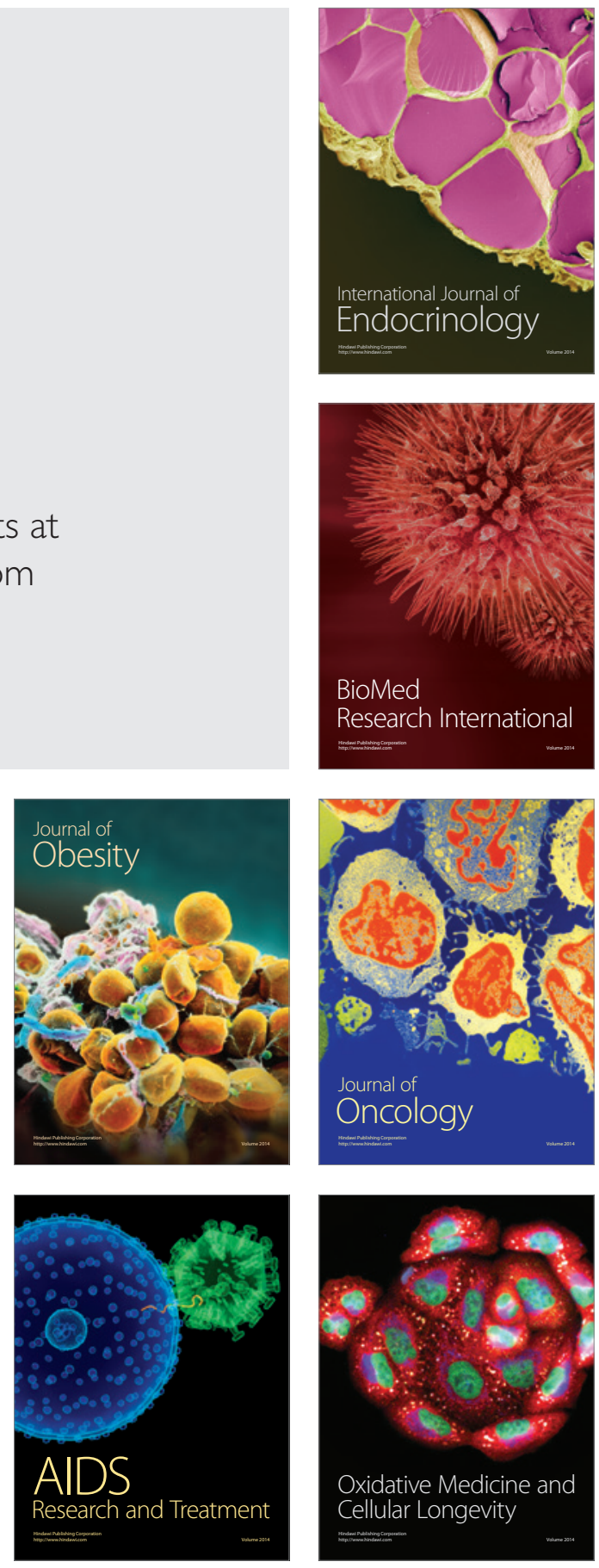